\title{
A Novel High Voltage Dielectric Test System Based on Resonant Circuits Using the Magnetically Controllable Inductance
}

\author{
Ying ZHANG ${ }^{1}$, Dangdang DAI ${ }^{2}$, Jing ZHANG ${ }^{3, *}$, Xuxuan $\mathrm{CHEN}^{4}$ \\ ${ }^{1}$ Electric Power Research Institute of Guizhou Power Grid Co., Ltd., China \\ ${ }^{2}$ Information and Communication Center, State Grid Hubei Electric Power Co., Ltd, China \\ ${ }^{3}$ School of Electrical Engineering, Guizhou University, China \\ ${ }^{4}$ School of Information Science and Engineering, Wuhan University of Science and Technology, China \\ zhangjing@gzu.edu.cn
}

\begin{abstract}
Dielectric tests such as high voltage withstand test is important to verify whether the electrical devices are in reliable working condition or not. This paper presents a novel high voltage dielectric test system up to $160 \mathrm{kV}$ AC based on resonant circuits using the magnetically controllable inductance. Firstly, the working principle and the design of the magnetically controllable inductance are introduced. In addition, a finite-element model is completed and analyzed to verify the designs of the magnetically controllable inductance. Next, resonant circuits and the control scheme of the dielectric test system are described. Finally, the proposed testing system is simulated using PSCAD/EMTDC. The test system can perform series or parallel resonance $A C$ tests by adjusting the inductance in the resonant circuit to meet the various requirements of the equipment to be examined. Compared with the conventional high-voltage test systems, the proposed test system has the advantages of compact structure, stable output voltage, and strong adaptability of the test method.
\end{abstract}

Index Terms-high-voltage techniques, resonance, insulation testing, magnetic variables control, EMTDC.

\section{INTRODUCTION}

High voltage can lead to dielectric deterioration as soon as the electrical equipment is installed and operational. If the deterioration is not checked, the insulators in these devices may break down during some severe electrical failure, which can lead to utility malfunctions [1]. Thus, the dielectric condition of these devices must be regularly checked. This so-called electrical preventive maintenance and testing should have the ability to recognize the deterioration level and provide suggestions to fix the insulation problems.

There are many important electrical properties of the insulating materials that can be derived from high voltage tests, such as AC/DC resistance, AC dielectric loss, DC dielectric absorption, and dielectric strength. Among the electrical properties, the dielectric strength can only be measured from destructive tests to check the correct manufacture of high voltage insulation. The dielectric strength check must be completed in both routine tests and on-site tests [2],[3]. As one of the most significant tests, onsite high voltage withstand tests are to quantify the

This work was supported by the Science and Technology Project of China Southern Power Grid Co., Ltd. under Grant No. GZKJXM20160018; the National Natural Science Foundation of China under Grant No. 51867005 . insulation quality of the electrical equipment [4],[5].

Several voltage shapes are used for different test purposes in the on-site tests. However, the alternating voltages generated by the on-site testing systems are the most important. When an $\mathrm{AC}$ voltage is applied to insulation materials, the currents drawn by the insulation are mainly due to capacitance charging, dielectric absorption and continuous leakage current. The measured current can clearly reflect the insulation status of the devices. For most electrical devices whose rated voltage is up to $110 \mathrm{kV}$, the equivalent capacitances are below $10000 \mathrm{pF}$, and the test current flowing into the insulators is very small. This means a normal transformer with high rated power is not required in the dielectric test system. As the core component of the test system, the transformer is used to output high voltage; but it is not required to output high current so that the volume of the whole test system can be compact [7]. However, it is difficult to meet the test requirements when performing a test on large generators, power transformers, cables, or GIS, because these devices generate large capacitive current which will surpass the power of a normal test system [8-10]. In such circumstances, a resonant test circuit can solve the problem [11].

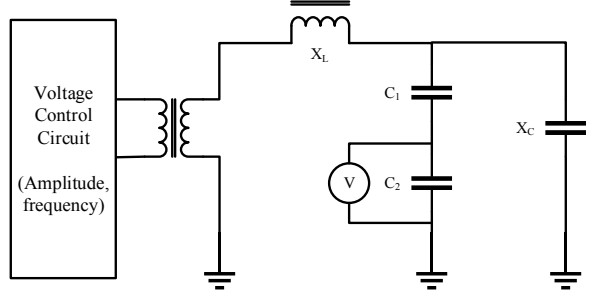

(a) Series resonant test circuit

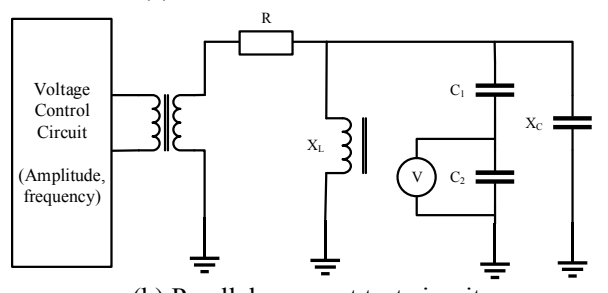

(b) Parallel resonant test circuit

Figure 1. Conventional high voltage withstand test system circuits

The resonant test circuit can be series or parallel resonant. The circuits for tests are shown in Fig. 1. If the output current of the transformers in the test system is satisfied with the test target devices, but the output current is below 
the withstand test voltage, the series resonant circuit can be used [12-18]. If the output voltage of the transformers is above the withstand test voltage, but the output current is lower than the requirement, the parallel resonant circuit is suggested [19-25]. The test system mainly consists of four parts including a voltage source, a transformer, a resonant inductance and a capacitor voltage transformer. The amplitude and the frequency of the voltage source can be regulated. However, since the resonant inductance is of a fixed value and the frequency tuning range of the source is normally limited between $30 \mathrm{~Hz}$ and $300 \mathrm{~Hz}$, the dielectric test will not get the required voltage if the equivalent capacitor of the target devices is out of resonant. Therefore, for different test objects, the parameters of the test system must match with each other. Considering that the circuits of the dielectric test system shown in Fig. 1 is also unchangeable after the design is complete, it is especially not convenient when there are lots of devices to be tested yearly in electrical preventive maintenance.

This paper proposed a resonant circuit based on the magnetically controllable inductance (MCI). The inductance of the MCI can be regulated smoothly, so that the resonant circuits can generate a wide output voltage range with the same voltage control source [26]. Besides, the MCI can work on either series or parallel with the test target by simply adding switches in the circuits. By tuning both the inductance of the MCI and the voltage source in the control system, both the voltage and the current for the test can meet with the due requirements.

The rest of the paper is organized as follows:

In section II, the working principle of the proposed dielectric test system is introduced. In section III, as the key component in the test system, the MCI is fully described. Section IV describes the control of the MCI inductance and the dielectric test system. Finally, a PSCAD/EMTDC model is built to simulate the voltage withstand test system. The model is used to verify if the proposed system can be used to meet various test requirements. Section V presents a conclusion of the work.

\section{BASIC WorkING PRINCIPLE OF THE PROPOSED DIELECTRIC TEST SYSTEM}

The proposed dielectric test system can be digitally controlled, and the control system consists of two parts: the voltage control source and the MCI. The rest components of the test system are the same as the conventional ones. The proposed dielectric test system based on the MCI is shown in Fig. 2.

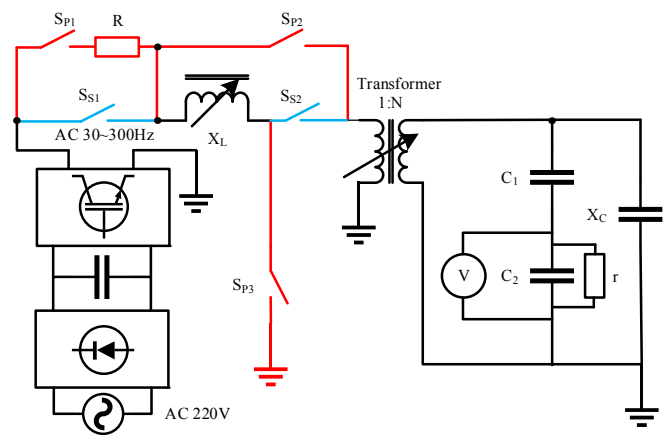

Figure 2. Proposed dielectric test system based on MCI

As shown in Fig.2, several switches are added in the proposed resonant circuit. The switches can be used to transfer the MCI from a series resonant circuit to a parallel resonant circuit. The circuit of the test system is in series resonant when $\mathrm{S}_{\mathrm{S} 1}, \mathrm{~S}_{\mathrm{S} 2}$ are on and $\mathrm{S}_{\mathrm{P} 1}, \mathrm{~S}_{\mathrm{P} 2}, \mathrm{~S}_{\mathrm{P} 3}$ are off, or in parallel resonant when $\mathrm{S}_{\mathrm{S} 1}, \mathrm{~S}_{\mathrm{S} 2}$ are off and $\mathrm{S}_{\mathrm{P} 1}, \mathrm{~S}_{\mathrm{P} 2}, \mathrm{~S}_{\mathrm{P} 3}$ are on.

In most situations, there is no need to control the output frequency in the voltage control source. The test system can output the required voltage by adjusting the boosting transformer and tuning the reactance $\mathrm{X}_{\mathrm{L}}$ of the MCI. If the required current or voltage output is higher, the output frequency of the voltage control source needs to be regulated so as to stimulate the voltage generated in the resonant circuit.

In the proposed test system, the transformer and the MCI can share a common magnetic circuit that can greatly reduce the volume of the whole system.

\section{A. Measurement circuit}

The output voltage is measured from the capacitor divider. If $C_{2} \gg C_{1}$, the measured voltage will be,

$$
U_{C_{2}}=\frac{C_{1}}{C_{1}+C_{2}} U_{X_{C}} \approx \frac{C_{1}}{C_{2}} U_{X_{C}}
$$

In the divider, there is a resistor in parallel with capacitor $C_{2}$ which can eliminate the residual charge after the measurement is done.

$$
r \gg 1 / \omega C_{2}
$$

The time constant of the parallel circuit consists of $C_{2}$ and $r$ is around 1 to 2 seconds.

\section{B. Series resonant circuit}

If the output voltage is below the requirement of the test, the circuit should be series resonant. When $N^{2} X_{L}$ equals $X_{C}$, the circuit will output its maximum voltage. The capacitors in the measurement circuit can be neglected. The output voltage can be present as,

$$
U_{X_{C}}=N U_{X_{L}}=N I X_{L}=N \frac{U_{1}}{R_{L}} X_{L} \approx U_{2} \frac{X_{L}}{R_{L}}
$$

where $U_{1}$ is the output voltage of the source, $U_{2}$ is the secondary voltage of the transformer, and $R_{\mathrm{L}}$ is the internal resistor of the MCI.

The value of $X_{\mathrm{L}} / R_{\mathrm{L}}$ is the quality factor of the MCI. It will be easy to get the required test voltage if a larger quality factor of the MCI is chosen. The output voltage will be $X_{\mathrm{L}} / R_{\mathrm{L}}$ times the secondary voltage of the regulator transformer.

\section{Parallel resonant circuit}

If the output voltage can meet the test requirement, but the output current is low, the circuit should be parallel resonant. In such circumstances, there will be theoretically infinite current flowing into the measurement circuit and the test target. Because there is no inductance in series with the first winding of the transformer, a small resistor $\mathrm{R}$ needs to be installed to limit the possible short circuit current.

The total capacitive output current $I_{\mathrm{C}}$ is,

$$
I_{C} \approx 2 \pi f C_{x} U
$$

where $U$ is the secondary voltage of the transformer, $f$ is the tuning frequency of the voltage control source, and $C$ is the total capacitor value of the test target and the measurement 
circuit.

By adjusting the value of $X_{\mathrm{L}}$, if the compensating current $I_{\mathrm{XL}} / \mathrm{N}$ is close to $I_{\mathrm{C}}$, the current flow in the first winding of the transformer will be small so that a large capacity transformer is not needed in the test system.

\section{THE MODEL OF THE MCI}

As the core component in the proposed system, the MCI is a specially designed inductor in which the iron cores are deeply saturated during the regulation. The magnetic flux density is usually higher than $1.8 \mathrm{~T}$. By controlling the saturation degree of the ferromagnetic material, the inductance of the MCI can be smoothly regulated. The equivalent circuit of the MCI is shown in Fig. 3.

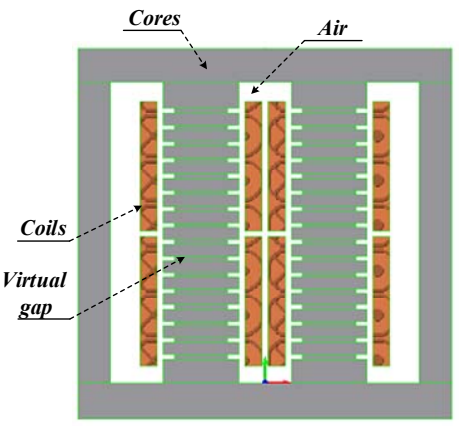

(a) Structure of the MCI in 2D

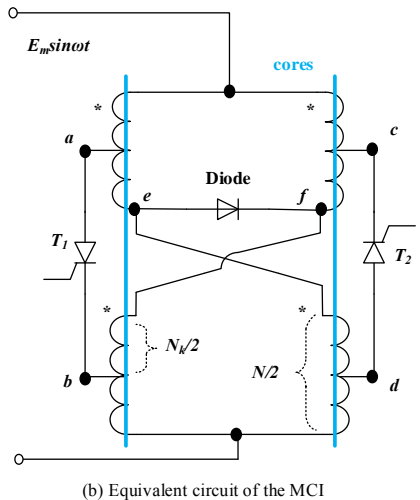

Figure 3. Structure and equivalent circuit of the MCI

\section{A. Coil circuit of the MCI}

Unlike normal inductors of iron core type, the iron core of the MCI has an additional limb in its center. There are also several virtual gaps filled in the limbs. The material of the virtual gaps is the same as that of the main cores. However, the ferromagnetic material in the gaps will quickly turn into saturation when DC current is injected into the coils. If the saturation degree in these gaps is high enough, the magnetic permeability is close to the air.

The DC current injected into the coils may be generated by full-wave or half-wave rectifiers. As shown in Fig. 3(b), a rectifier with two thyristors is used to generate a stable DC current in the coils. The thyristors connect the taps in the coils so that no additional AC source is needed. The turn number of the coupling coil $N_{\mathrm{k}}$ is $5 \%$ of the total turn number of the main coil attached to each limb. The benefit of this setup is obvious. When voltage amplitude $E_{\mathrm{m}}$ is high, the voltage on the thyristors are low. Therefore, there is no need to connect several thyristors in series connection to share the overvoltage risk.

When the operating voltage is positive and thyristor $\mathrm{T} 1$ is conducted, points $\mathrm{a}$ and $\mathrm{b}$ are short circuit. The coil voltages between points $\mathrm{fb}$ and $\mathrm{cf}$ are the same,

$$
u_{f b}=u_{c f}=\frac{N_{k}}{N} E_{m} \sin \omega t
$$

where $E_{\mathrm{m}} \sin \omega t$ is the operating voltage of the MCI.

The iron cores and coils are entirely symmetrical. It means that $u_{\text {be }}, u_{\text {ed }}, u_{\mathrm{fb}}, u_{\mathrm{cf}}$ have the same voltage value.

The commutation circuits of the MCI when thyristors are conducted are shown in Fig. 4.
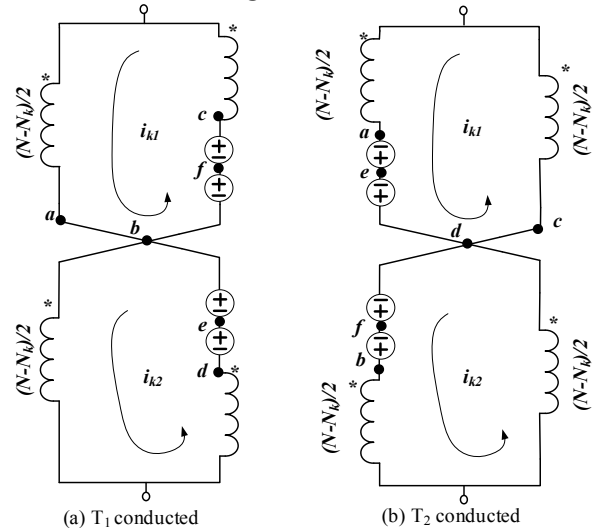

Figure 4. The commutation circuits of the MCI when thyristors are conducted

As shown in Fig.4(a), since thyristors can only be conducted in one direction, there will be a DC current flows along the paths $a, b, f, c$ and get back to point $a$.

The circuit analysis is the same when the operation voltage is negative and thyristor $T_{2}$ is conducted. As shown in Fig.4(b), points $c$ and $d$ are short, and the coil voltage between point $a e, e d, d f$ and $f b$ are all the same.

The DC currents $i_{\mathrm{k} 1}, i_{\mathrm{k} 2}$ will saturate the ferromagnetic material in the iron cores. The values of $i_{\mathrm{k} 1}, i_{\mathrm{k} 2}$ can be controlled by changing the fire angles of the thyristors.

\section{B. Magnetic behaviors in the iron cores}

The inductance of the MCI is very large when there is no DC current in the coils. However, the MCI inductance will soon decrease when the iron cores are saturated.

Normally, the output current of a conventional inductor contains great harmful harmonics if the iron cores are saturated. However, not every part of the iron cores in the MCI is statured. Only those virtual gaps with smaller areas will be deeply saturated. The magnetic permeability of the virtual gaps is close to the air if deeply saturated.

There are several virtual gaps of different area values, so the magnetic flux density in these virtual gaps is also different. Considering that the saturation degree has a great impact on the equalized magnetic permeability of the gaps, the actual gap length in the iron cores can be indirectly regulated by the injection of controlled DC magnetic flux components.

The structure of the virtual gap in the iron core is shown in Fig. 5. $A_{\mathrm{b}}, A_{\mathrm{s} 1}$ and $A_{\mathrm{s} 2}$ are the areas of the iron core, the first virtual gap with the smallest area, and second virtual gap, respectively. In the actual MCI production process, the silicon steel sheets are made as shown in the upper figure of Fig.5(b) to reduce the flux leakage of the virtual gaps when they are saturated.

Define $B_{\mathrm{ts}}$ as the magnetic flux density when the iron cores with area $A_{\mathrm{b}}$ begin to saturate. Then, the magnetic flux density when the first and second virtual gaps begin to 
saturate can be described as,

$$
\begin{aligned}
& B_{t 1}=\frac{A_{s 1}}{A_{b}} B_{t s} \\
& B_{t 2}=\frac{A_{s 2}}{A_{b}} B_{t s}
\end{aligned}
$$

The value of $B_{\mathrm{ts}}$ is usually set to $1.8 \mathrm{~T}$ for silicon steel material.
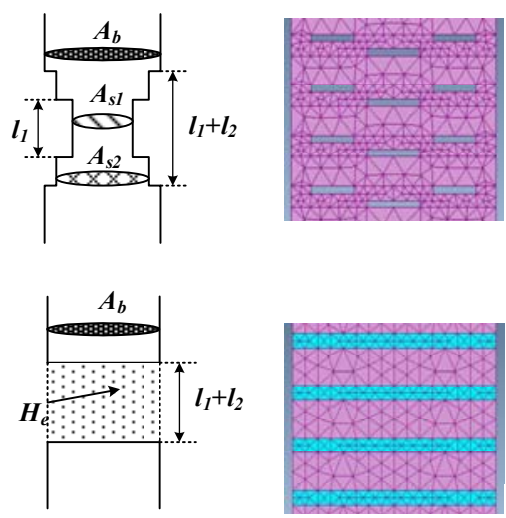

(a) Virtual gap in the MCI (b) 2D Mesh in FEM software

Figure 5. Structure of the virtual gap in the iron core

Take the first virtual gap as an example. According to the magnetic flux continuity low, the magnetic flux in the iron cores is,

$$
\Phi=B A_{b}=B_{0}\left(A_{b}-A_{s 1}\right)+B_{s 1} A_{s 1}
$$

where $B$ is the magnetic flux density in the iron core, $B_{0}$ is the magnetic flux density in the air gap, and $B_{\mathrm{s} 1}$ is the magnetic flux density in the first stage.

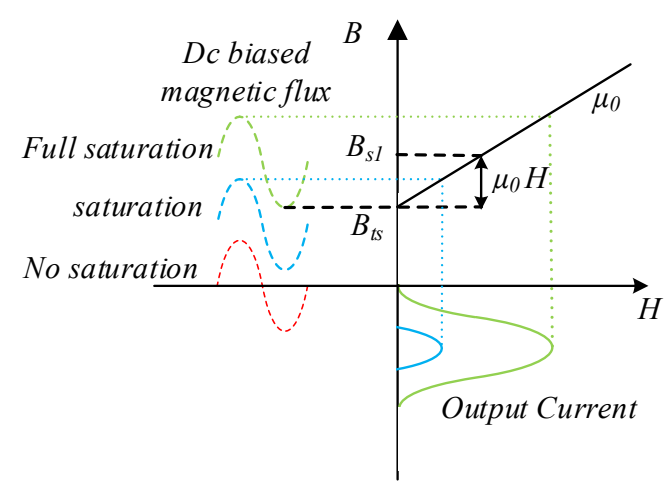

Figure 6. BH characteristics when iron cores are in Deep saturation

The $\mathrm{BH}$ characteristics when iron cores are in deep saturation is shown in Fig. 6. Since $B_{\mathrm{ts}}$ value is the magnetic density when the silicon steel material is saturated, the $\mathrm{BH}$ behavior is quite different from that in the normal transformer cores. The slope of the line nearly equals the magnetic permeability value of the air $\mu_{0}$. Thus, the relative value of $\mathrm{H}$ is much higher.

As shown in Fig. 6, the magnetic flux in the iron cores can reflect the output current by mapping based on the simplified liner function of the BH characteristics. If there is no DC current in the coils, the output current equals zero. When DC current increases as the firing angle changes, the output current begins to increase. However, the output current is a non-sinusoidal wave which contains harmonics. It will become a sinusoidal wave completely until the ferromagnetic material is fully saturated.

Therefore, when there are any DC currents in the coils, the smallest virtual gap will soon be driven into saturation. And, the analytical method is the same when $\mathrm{BH}$ is negative. From equation (8), the magnetic flux density in the iron core $B$ can be described as,

$$
\begin{aligned}
B & =B_{0} \frac{A_{b}-A_{s 1}}{A_{b}}+B_{s 1} \frac{A_{s 1}}{A_{b}} \\
& =\mu_{0} H \frac{A_{b}-A_{s 1}}{A_{b}}+\left(\mu_{0} H+B_{t s}\right) \frac{A_{s 1}}{A_{b}}
\end{aligned}
$$

Because the currents flow in the coils are the same, according to the Ampere's circuit law,

$$
H_{e}\left(l_{1}+l_{2}\right)=H_{1} l_{1}=\sum I
$$

Then, $H_{\mathrm{e}}$ can be rewritten as,

$$
H_{e}=\frac{B-\frac{A_{s 1}}{A_{b}} B_{t s}}{\mu_{0}} \cdot \frac{l_{1}}{l_{1}+l_{2}}
$$

Similarly, when the second virtual gap begins to saturate, $H_{\mathrm{e}}$ is given as,

$$
H_{e}=\frac{B-\frac{A_{s 1}}{A_{b}} B_{t s} \frac{l_{1}}{l_{1}+l_{2}}-\frac{A_{s 2}}{A_{b}} B_{t s} \frac{l_{2}}{l_{1}+l_{2}}}{\mu_{0}}
$$

Normalize the lengths as $L_{\mathrm{t} 1}=l_{1} /\left(l_{1}+l_{2}\right), L_{\mathrm{t} 2}=l_{2} /\left(l_{1}+l_{2}\right)$. By substitute equations (6) and (7) into equation (12), the $\mathrm{BH}$ characteristics of the virtual gaps can be written as

$$
H_{e}=f(B)= \begin{cases}0 & |B|<B_{t 1} \\ \frac{B-B_{t 1}}{\mu_{0}} L_{t 1} & B_{t 1} \leq B<B_{t 2} \\ \frac{B-B_{t 1} L_{t 1}-B_{t 2} L_{t 2}}{\mu_{0}} & B \geq B_{t 2} \\ \frac{B+B_{t 1}}{\mu_{0}} L_{t 1} & -B_{t 2}<B \leq-B_{t 1} \\ \frac{B+B_{t 1} L_{t 1}+B_{t 2} L_{t 2}}{\mu_{0}} & B \leq-B_{t 2}\end{cases}
$$

From equation (13), the $\mathrm{BH}$ characteristics of the virtual gaps can be obtained.

\section{The output current of the MCI}

As the key component of the dielectric test system, the MCI will output current which contains several harmonics components. Since THD of the output current seriously affects the regulation accuracy of the MCI inductance, the circuit may not achieve resonance if there are excessive harmonics. So it is required that the THD of the output should be optimized

The magnetic flux densities in the right and left iron cores are $B_{1}$ and $B_{2}$, respectively. They will be biased in the opposite directions when there are DC currents in the coils. Define the DC bias magnetic flux density as $B_{\mathrm{d}}, B_{1}$ and $B_{2}$ can be described as,

$$
\left\{\begin{array}{l}
B_{1}(\omega t)=B_{d}+B_{1} \\
B_{2}(\omega t)=-B_{d}+B_{2}
\end{array}\right.
$$

To describe the saturation degree quantitatively, $\beta_{1}$ and $\beta_{2}$ are introduced. They represent the saturation degrees of the first and the second virtual gaps in a power frequency. The definition is shown in Fig. 7. 
When $B_{d}=0$, the virtual gaps are not saturated, $\beta_{1}$ and $\beta_{2}$ are given by

$$
\left\{\begin{array}{l}
\beta_{1}=0 \\
\beta_{2}=0
\end{array} \quad B \leq B_{t 1}\right.
$$

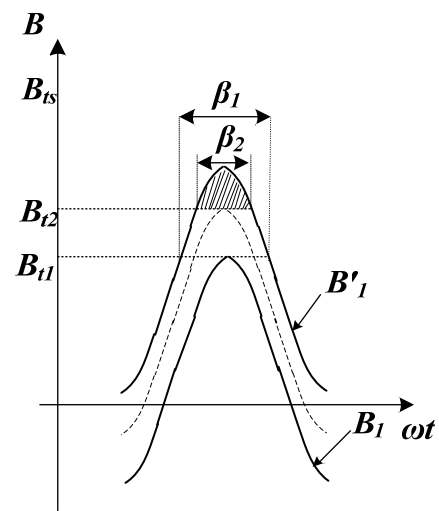

Figure 7. Saturation degrees calculation of the virtual gaps

When the first virtual gap begins to saturate and the second virtual gap remains unsaturated, the saturation degrees are,

$$
\left\{\begin{array}{l}
\beta_{1}=2 \cos ^{-1} \frac{B_{t 1}-B_{d}}{B_{t 1}} \\
\beta_{2}=0
\end{array} B_{t 1}<B \leq B_{t 2}\right.
$$

When the virtual gaps are both saturated,

$$
\left\{\begin{array}{l}
\beta_{1}=2 \cos ^{-1} \frac{B_{t 1}-B_{d}}{B_{t 1}} \\
\beta_{2}=2 \cos ^{-1} \frac{B_{t 2}-B_{d}}{B_{t 1}}
\end{array} \quad B_{t 2} \leq B \leq B_{t s}\right.
$$

From (17), the relationship between $\beta_{1}$ and $\beta_{2}$ can be written as

$$
\beta_{2}=2 \cos ^{-1}\left(\frac{B_{t 2}}{B_{t 1}}-1+\cos \frac{\beta_{1}}{2}\right)
$$

Define $S=B_{t 2} / B_{t 1}$, from equations (6) and (7), $S$ can be given as

$$
S=\frac{B_{t 2}}{B_{t 1}}=\frac{A_{s 2} / A_{b}}{A_{s 1} / A_{b}}=\frac{A_{s 2}}{A_{s 1}}
$$

From equation (19), $S$ equals the area ratio between the second and the smallest virtual air gap. Considering that the iron cores cannot be saturated during the regulation, the maximal $\beta_{1}$ can be given as

$$
\beta_{\max }=2 \cos ^{-1}\left(2-\frac{A_{b}}{A_{s 1}}\right)
$$

In equation (20), $\beta_{\max }$ value is $2 \pi$ when $A_{\mathrm{b}} / A_{\mathrm{s} 1}=3$. Since $A_{\mathrm{s} 2}$ value is between the values of $A_{\mathrm{s} 1}$ and $A_{\mathrm{b}}$, in equation (19), the value of $S$ is between 1 and 3 .

$B_{1}$ and $B_{2}$ are the magnetic flux density in the left and right iron cores. From the simplified electrical circuit, the magnetic formula for MCI is derived as,

$$
\left\{\begin{array}{l}
e=E_{m} \sin \omega t=N A_{b}\left(\frac{d B_{1}}{d t}+\frac{d B_{2}}{d t}\right) \\
E_{k}=R_{k} i_{k}+N_{k} A_{b}\left(\frac{d B_{1}}{d t}-\frac{d B_{2}}{d t}\right) \\
N i+N_{k} i_{k}=l \cdot H_{e 1} \\
N i-N_{k} i_{k}=l \cdot H_{e 2} \\
H_{e 1}=f\left(B_{1}\right) \\
H_{e 2}=f\left(B_{2}\right)
\end{array}\right.
$$

In equation (21), via FFT analysis, the magnetic flux density $B_{1}$ and $B_{2}$ can be expressed as,

$$
\left\{\begin{array}{l}
B_{1}(\omega t)=B_{d}+\sum_{k=1}^{n} B_{(k) m} \sin \left(k \omega t-\phi_{k}\right) \\
B_{2}(\omega t)=-B_{d}+\sum_{k=1}^{n} B_{(k) m} \sin \left(k \omega t-\phi_{k}\right)
\end{array}\right.
$$

where $k(1,2,3 \ldots)$ is the order of the specified harmonic.

Considering that $B_{1}$ and $B_{2}$ are both periodic,

$$
\left\{\begin{array}{l}
B_{1}(\omega t)=-B_{2}(\omega t+\pi) \\
B_{2}(\omega t)=-B_{1}(\omega t+\pi)
\end{array}\right.
$$

By substituting equations (22) and (23) into equation (21),

$$
\left\{\begin{array}{l}
e=\omega N A_{b} \sum_{k=1}^{n}(2 k-1) B_{(2 k-1) m} \cos \left[(2 k-1) \omega t-\phi_{2 k-1}\right] \\
E_{k}=\omega N A_{b} \sum_{k=1}^{n} 4 k B_{(4 k) m} \cos \left(2 k \omega t-\phi_{2 k}\right)
\end{array}\right.
$$

From equation (21), the output current $i$ is,

$$
i=\frac{l}{2 N}\left[f\left(B_{1}\right)+f\left(B_{2}\right)\right]
$$

Since the $\mathrm{BH}$ characteristics is an odd function, from equation (23),

$$
\begin{aligned}
f\left[B_{1}(\omega t)\right] & =f\left[-B_{2}(\omega t+\pi)\right] \\
& =-f\left[B_{2}(\omega t+\pi)\right]
\end{aligned}
$$

From the above equations, define the reference value as $B_{t l} l / N \mu_{0}$, the nominal fundamental current is,

$$
i_{1}^{*}=\frac{1}{2 \pi}\left[\left(\beta_{1}-\sin \beta_{1}\right) L_{t 1}+\left(\beta_{2}-\sin \beta_{2}\right) L_{t 2}\right]
$$

And the nominal current harmonics can be described as,

$$
\begin{aligned}
i_{2 k+1}^{*}= & \frac{L_{t 1}}{(2 k+1) \pi}\left\{\frac{\sin \left(k \beta_{1}\right)}{2 k}-\frac{\sin \left[(k+1) \beta_{1}\right]}{2(k+1)}\right\}+ \\
& \frac{L_{t 2}}{(2 k+1) \pi}\left\{\frac{\sin \left(k \beta_{2}\right)}{2 n}-\frac{\sin \left((k+1) \beta_{2}\right)}{2(k+1)}\right\}
\end{aligned}
$$

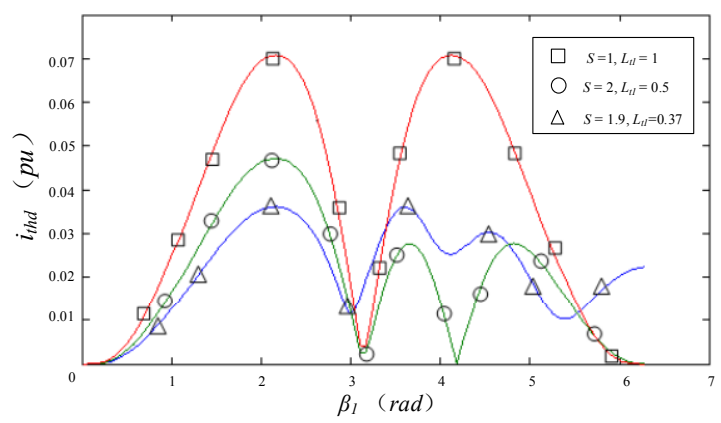

Figure 8. THD of the output current versus the saturation degree of the smallest virtual gap

The THD of the output current versus the saturation degree $\beta_{l}$ of the smallest virtual gap is shown in Fig. 8 when area ratio $S$ and normalized lengths ratio $L_{\mathrm{t} 1}$ have different 
values. The optimal maximum THD value (blue line) is $3.6 \%$ when $S=1.9$ and $L_{\mathrm{t} 1}=0.37$.

\section{Simulation of the Dielectric Test System}

The proposed voltage withstand test system is a compact structure that can be manufactured by combining the MCI and the transformer together using the same magnetic flux path. It means that both the MCI and the transformer can use the same iron cores. Since the rated power of the transformer (80kVA) is much larger than the MCI (10kVA), the volume and weight of the whole test device are mainly determined by the transformer.

The control strategy is simple. At fixed frequency $50 \mathrm{~Hz}$, the generated voltage can be regulated by controlling only the inductance of the MCI. Otherwise, if the output voltage or current cannot meet the test requirements during the regulation, it is necessary to tune the frequency of the test source to set the circuit resonant. In extreme situations, both the frequency and the inductance of the MCI need control.

\section{A. Inductance control of the MCI}

The electrical circuit of the MCI is shown in Fig. 9. $R$ and $R_{k}$ are the DC resistors of the main coil and the coupling coil, respectively.

The commutation circuits of the MCI shown in Fig.4 indicate that the two thyristors work as a full rectifier. Thus, the rectifier can be simplified as a controllable DC-bias source, $\mathrm{E}_{\mathrm{k}}$.

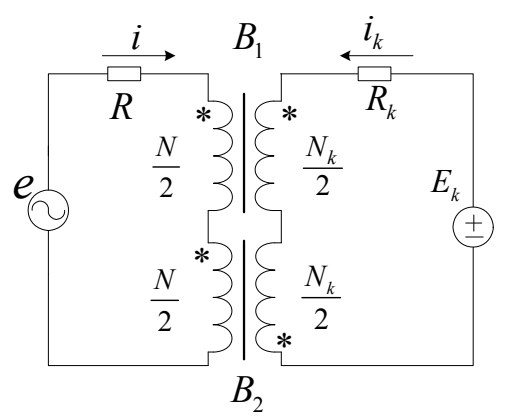

Figure 9. The simplified electrical circuit of the MCI

From Fig. 9, the simulation circuit of the MCI can be easily modeled by using two saturable transformers. The UMEC transformers model is needed to simulate the saturation behavior of the MCI in PSCAD/EMTDC. The controllable source $E_{k}$ can be equally modeled using 4 thyristors instead of two. The model of the MCI is shown in

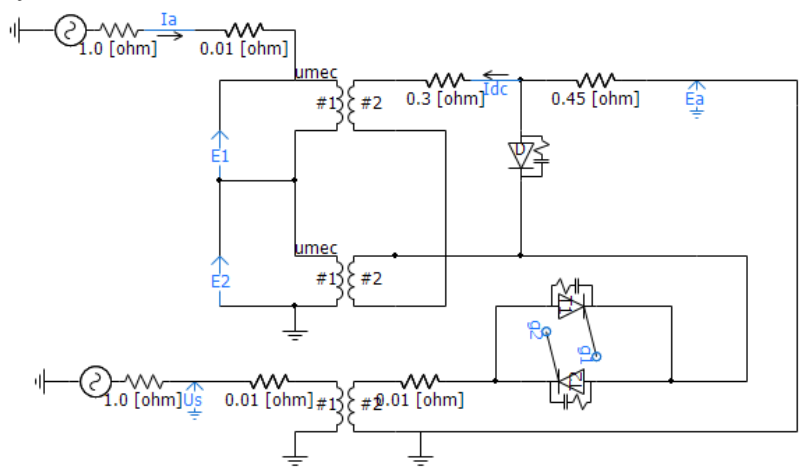

Figure 10. Simulation model of the MCI in PSCAD/EMTDC

The model of two UMEC transformers are the same. From equation (13), the equalized $\mathrm{BH}$ characteristics of the main core can be derived. The result is listed in Table. I.

TABLE I. THE EQUALIZED BH CHARACTERISTIC OF THE IRON CORES

\begin{tabular}{|c|c|c|}
\hline Point & Current (\%) & Voltage (p.u.) \\
\hline 1 & 0 & 0 \\
\hline 2 & 0.1 & 1 \\
\hline 3 & 33 & 1.9 \\
\hline 4 & 143 & 3 \\
\hline 5 & 243 & 4 \\
\hline
\end{tabular}

The primary voltage and secondary voltage of the $10 \mathrm{kVA}$ UMEC transformers are both $220 \mathrm{~V}$, which is half of the source voltage of the utility voltage. The output current of the MCI is shown in Fig. 11. The highest RMS value of the output current is $21.76 \mathrm{~A}$. It means that the inductance of the MCI can be smoothly regulated from a high inductive reactance to as small as $20.22 \Omega$ at $50 \mathrm{~Hz}(64 \mathrm{mH})$.

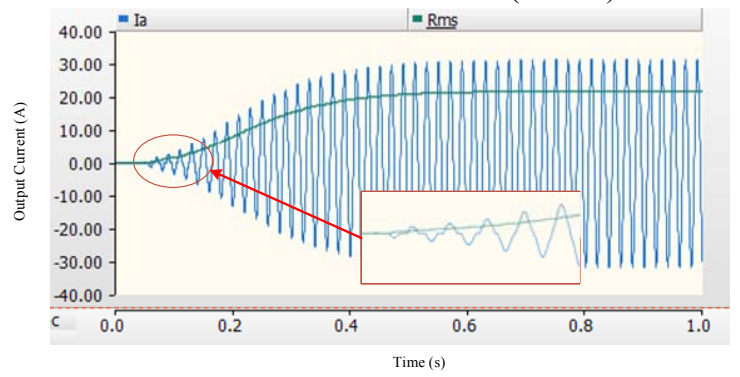

Figure 11. Output current and the RMS value of the MCI

The magnetic flux waveforms are shown in Fig.12 when the MCI outputs the rated current.

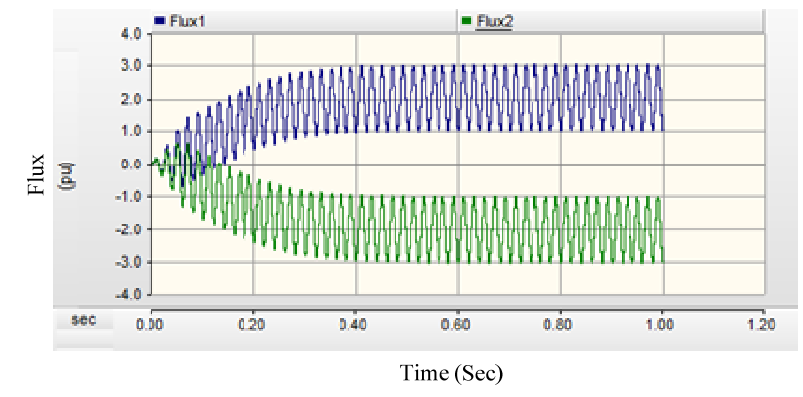

Figure 12. The flux waveforms of the UMEC transformers

The results agree with the theoretical analysis shown in Fig. 6. The FEM analysis of the MCI in a power period is shown in Fig. 13 when the left or right core is saturated.

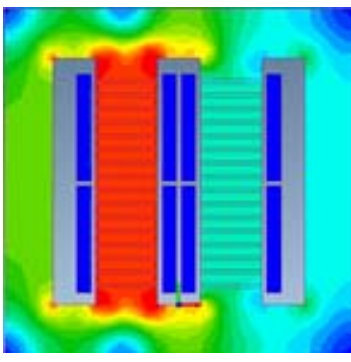

(a) Saturated left core

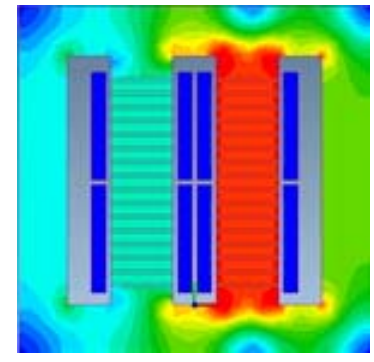

(b) Saturated right core
Figure 13. FEM analysis of the MCI in a power period

Since the output current versus the input voltage of the MCI is linear, the inductance of the MCI is mainly related to the DC excitation current. The control scheme is shown in Fig. 14 when the voltage upon the MCI is rated. 


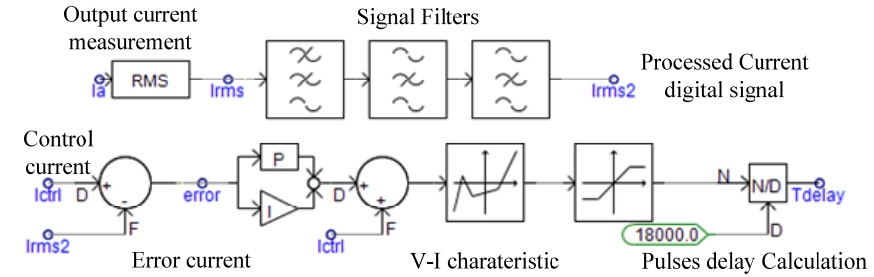

Figure 14. Inductance control of the MCI

For specified inductance of the MCI, the output current can be calculated using Ohm's law. However, the firing angle of the thyristors versus the output current is nonlinear. The relationship can be obtained by the test shown in Table II. The output current has good linear performance when the firing angle is between 40 and 140 degrees.

TABLE II. THE OUtPut CURRENTS WHEN FIRING ANGLES ARE DiFFERENT

\begin{tabular}{|c|c|c|}
\hline Point & Current (A) & Firing Angle $^{\circ}{ }^{\circ}$ ) \\
\hline 1 & 0 & 180 \\
\hline 2 & 0.5 & 160 \\
\hline 3 & 2.47 & 140 \\
\hline 4 & 6.99 & 120 \\
\hline 5 & 12.01 & 100 \\
\hline 6 & 16.32 & 80 \\
\hline 7 & 19.06 & 60 \\
\hline 8 & 20.89 & 40 \\
\hline 9 & 21.75 & 20 \\
\hline 10 & 21.76 & 0 \\
\hline
\end{tabular}

Via Table. II, the pulses to trig the thyristors can be got and turn into time delay signals which are used in the timebased thyristors control model of PSCAD/EMTDC. The firing angle signals are finally connected to g1 to g4 which are shown in Fig. 10.

\section{B. Simulation of the proposed voltage withstand system}

The simulation circuit shown in Fig. 15 is the same as the theoretical circuit which is shown in Fig. 2.

The MCI is connected to the test circuit by a coupling magnetic circuit of which the turn ratio is 16 . The turn ratio between the rated primary and secondary voltages of the boost transformer is 1:40. The leakage of these transformers needs to be carefully designed so that overvoltage will not happen during the voltage regulation.

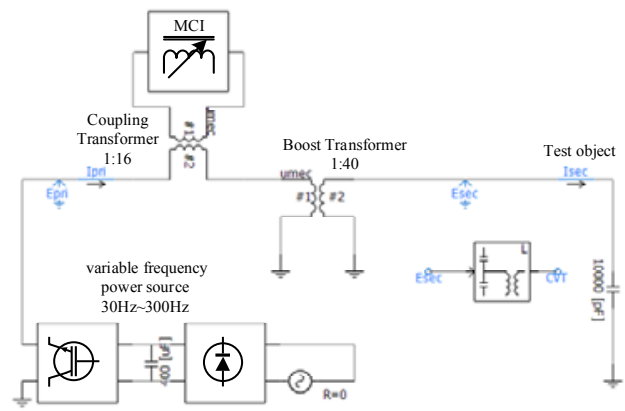

(a) series resonant circuit

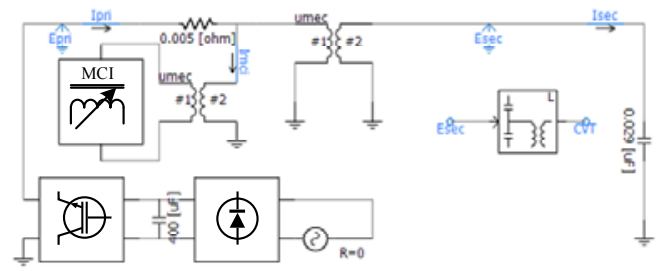

(b) parallel resonant test circuit

Figure 15. Simulation model of the dielectric test system
Take a series resonant circuit test system as an example, the VI characteristics of the voltage withstand system without test object are shown in Fig. 16. The curves show that the test system has a good linear characteristic.

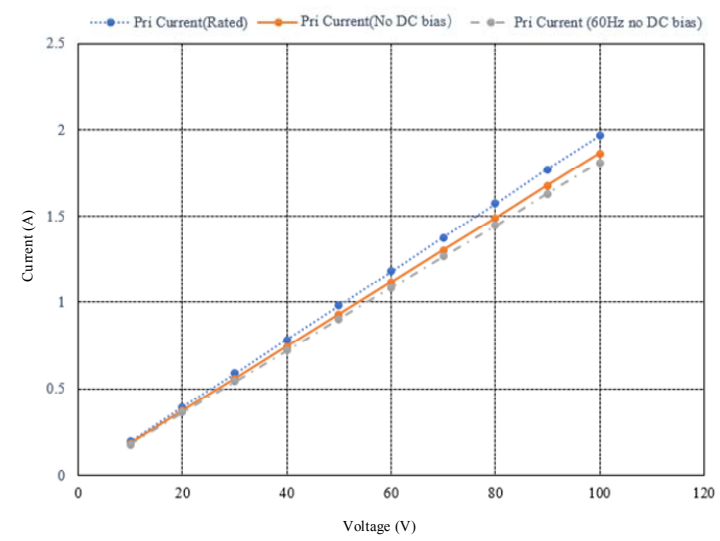

Figure 16. VI characteristics of the voltage withstand system without test objects

Simulation waveforms of the proposed voltage withstand system is shown in Fig. 17 when series resonant happens. The inductance the MCI deceases from $8 \mathrm{H}$ to $95 \mathrm{mH}$ at the time 0.3 second.

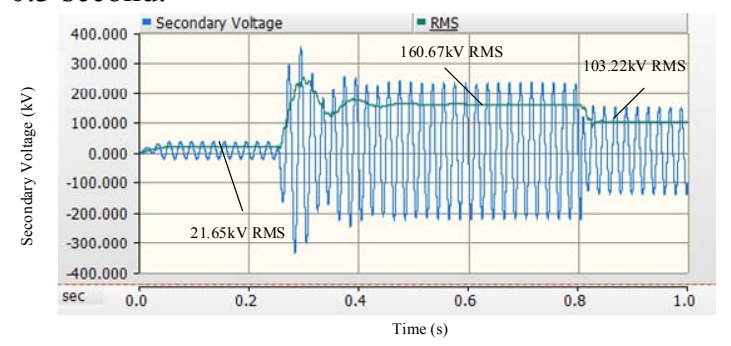

Figure 17. Simulation of the voltage withstand system (series resonant)

At the start of the test, increase the primary voltage to $380 \mathrm{~V}$ (peak value) gradually. The secondary voltage is $21.65 \mathrm{kV}$ when the inductance of $\mathrm{MCI}$ is $8 \mathrm{H}$. At the time 0.3 second, regulate the inductance to $95 \mathrm{mH}$, and the output voltage suddenly increases to $355.12 \mathrm{kV}$ (peak value) and soon decays to $160.67 \mathrm{kV}$ (RMS value). If the test object fails at the time 0.8 , the breaker operates immediately, and the voltage soon falls to $103 \mathrm{kV}$ RMS.

For a parallel resonant test system, the test object is a $110 \mathrm{kV} / 0.029 \mu \mathrm{F}$ capacitor. The test needs to add $80 \mathrm{kV}$ voltage to the capacitor which will also output $728 \mathrm{~mA}$ in the secondary winding. If there is no compensate inductor, a large capacitive source on the primary side of the transformer will be needed.

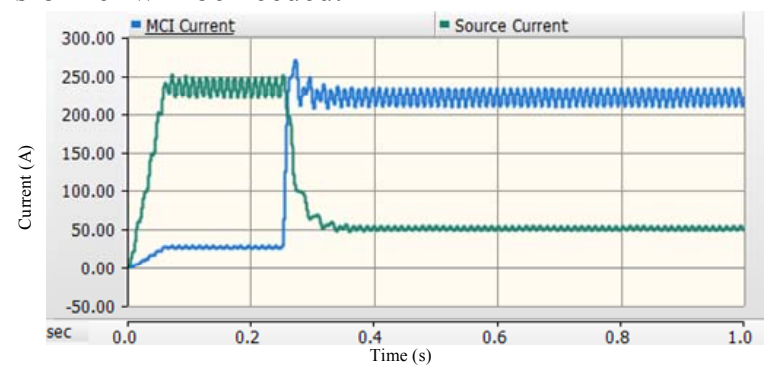

Figure 18. Simulation of the voltage withstand system (parallel resonant)

The simulation results are shown in Fig. 18. The inductor of the MCI changes from $8 \mathrm{H}$ to $64 \mathrm{mH}$ at the time 0.3 second. It compensates the capacitive current so that the current of 
the source decreases from $242 \mathrm{~A}$ to $50 \mathrm{~A}$. The source voltage is only $225 \mathrm{~V}$ (peak value) when the voltage is $80 \mathrm{kV}$ in the secondary winding. This means that only a source with $8 \mathrm{~kW}$ is needed.

\section{CONCLUSION}

This paper proposed a novel voltage dielectric test system based on the MCI. The MCI and boost transformer can be manufactured using the same iron cores. Because the rated power of the MCI is much lower, the volume and weight of the test device are mainly dependent on the transformer.

Compared to conventional test equipment, the compact test system can perform high voltage test using both series and parallel resonant methods in power-frequency.

The model of the proposed test system is described in detail and verified using the PSCAD/EMTDC. The results show that the proposed system is suitable for on-site high voltage ac insulation tests.

\section{REFERENCES}

[1] G. Xiangxiang, L. Haojun, J. Zhuanzhuan, and P. Jianfei, "Research of shunt reactor switching test for $1100 \mathrm{kV}$ UHV circuit-breakers," 2017 4th International Conference on Electric Power Equipment Switching Technology (ICEPE-ST), Xi'an, 2017, pp. 821-824. doi:10.1109/ICEPE-ST.2017.8188965

[2] C. Jingying, et al., "Study on Optimization of Withstand Voltage Test for Long-Distance Power Cable Multi-Resonance System," 2018 International Conference on Power System Technology (POWERCON), Guangzhou, 2018, pp. 3936-3939. doi:10.1109/POWERCON.2018.8601771

[3] Y. Li, J. Lian, J. Jin, Z. Liu, Z. Zhang and J. Cao, "Testing Research on Auxiliary Circuit Oscillation Characteristic Parameters for High Voltage DC Transfer Switch," 2018 IEEE 3rd Advanced Information Technology, Electronic and Automation Control Conference (IAEAC), Chongqing, 2018, pp. 1184-1188. doi:10.1109/IAEAC.2018.8577791

[4] W. Bo, et al., "Design of $1100 \mathrm{kV} / \mathrm{I0kA}$ Ultra-High-Voltage Alternating Current Long-term Live Test Loop," 2018 China International Conference on Electricity Distribution (CICED), Tianjin, 2018, pp. 685-692. doi:10.1109/CICED.2018.8592541

[5] C. Liu, M. Shen, Q. Li, C. Su, and P. Wei, "Application of Neural Network in Atmospheric Correction of High Voltage Test," 2018 IEEE International Conference on High Voltage Engineering and Application (ICHVE), ATHENS, Greece, 2018, pp. 1-4. doi:10.1109/ICHVE.2018.8642037

[6] G. Ueta, T. Tsuboi and S. Okabe, "Evaluation of overshoot rate of lightning impulse withstand voltage test waveform based on new base curve fitting methods - application to practical diverse waveforms," in IEEE Transactions on Dielectrics and Electrical Insulation, vol. 19, no. 1, pp. 352-362, February 2012. doi:10.1109/TDEI.2012.6148538

[7] H. Wang, Y. Cao, J. Fan, Research on Discharge Faults in DC Voltage Withstand Test of HVDC Project Converter Transformer, Transformer, vol. 56, no. 8, pp.60-62, August 2019.

[8] B. J. Lee, S. H. Kim, T. J. Kwon, J. H. Choi, and M. H. Kim, "Analysis of the test line impedance and strength for short time and peak withstand test of KERI's new high power laboratory," 2015 3rd International Conference on Electric Power Equipment - Switching Technology (ICEPE-ST), Busan, 2015, pp. 451-454. doi:10.1109/ICEPE-ST.2015.7368450

[9] S. X. Ma, et al., "Characteristics and application of the oscillating impulse voltage on GIS infield," 2018 12th International Conference on the Properties and Applications of Dielectric Materials (ICPADM), Xi'an, 2018, pp. 550-553. doi:10.1109/ICPADM.2018.8401096

[10] S. Jin, J. Ruan, Z. Du, R. Gong, and L. Zhu, "Research on corona control in DC voltage withstand test for UHV converter transformer," 2016 IEEE International Conference on High Voltage Engineering and Application (ICHVE), Chengdu, 2016, pp. 1-4 doi:10.1109/ICHVE.2016.7800627

[11] J. Yuan, et al., "A Novel Compact High-Voltage Test System Based on a Magnetically Controlled Resonant Transformer," in IEEE
Transactions on Magnetics, vol. 51, no. 11, pp. 1-4, Nov. 2015, Art no. 1700204. doi:10.1109/TMAG.2015.2449336

[12] L. Dong, J. Kong, J. Feng, and Y. Zhang, "Sub synchronous Resonance Mitigation for Series Compensation Transmission System of DFIG Based on PR Control," 2019 IEEE 10th International Symposium on Power Electronics for Distributed Generation Systems (PEDG), Xi'an, China, 2019, pp. 734-738. doi:10.1109/PEDG.2019.8807674

[13] N. Jahan, A. Barakat, and R. K. Pokharel, "Study of phase noise improvement of K-band VCO using additional series resonance realized by DGS resonator on CMOS technology," 2017 IEEE Asia Pacific Microwave Conference (APMC), Kuala Lumpur, 2017, pp 1014-1017. doi:10.1109/APMC.2017.8251624

[14] Y. Yang, R. Han, Y. Jin, R. Tao, T. Li, and N. Li, "Analysis of the Influencing Factors of Quality Factor of Series Resonance System with Large Capacity," 2019 IEEE 3rd International Conference on Circuits, Systems and Devices (ICCSD), Chengdu, China, 2019, pp. 65-68. doi:10.1109/ICCSD.2019.8843160

[15] W. Yeetum and V. Kinnares, "Parameters Identification for Series Resonance in Power Systems Using a Frequency Response Technique," 2018 International Electrical Engineering Congress (iEECON), Krabi, Thailand, 2018, pp. 1-4. doi:10.1109/IEECON.2018.8712295

[16] L. Liang et al., "Preliminary Experiment of a SFCL Based on AirCore Superconducting Transformer and Inductor-Capacitor Series Resonant Limiter," 2018 IEEE International Conference on Applied Superconductivity and Electromagnetic Devices (ASEMD), Tianjin, 2018, pp. 1-2. doi:10.1109/ASEMD.2018.8558996

[17] S. Wei, et al., "Analysis and Study of Resonance Monitoring and Suppression Strategies of EHV Main Transformer," 2016 3rd International Conference on Information Science and Control Engineering (ICISCE), Beijing, 2016, pp. 848-852. doi:10.1109/ICISCE.2016.185

[18] M. Lei, S. Wang, Y. Guo, T. Xia, D. Ming and X. Zheng, "An Automatic Implementation Scheme of in-Field Power Frequency Series Resonance System," 2018 2nd IEEE Conference on Energy Internet and Energy System Integration (EI2), Beijing, 2018, pp. 1-5. doi:10.1109/EI2.2018.8582377

[19] X. Zhang, C. Yu, F. Liu, F. Li, and H. Xu, "Overview on resonance characteristics and resonance suppression strategy of multi-parallel photovoltaic inverters," in Chinese Journal of Electrical Engineering, vol. 2, no. 1, pp. 40-51, June 2016. doi:10.23919/CJEE.2016.7933114

[20] S. Chaladying, A. Charlangsut, and N. Rugthaichareoncheep, "Parallel resonance impact on power factor improvement in power system with harmonic distortion," TENCON 2015 - 2015 IEEE Region 10 Conference, Macao, 2015, pp. 1-5. doi:10.1109/TENCON.2015.7373180

[21] Shengming Wang, Junfeng Chen, Zhaoyang Hu, and Minghai Liu, "Study on series-parallel mixed-resonance model of wireless power transfer via magnetic resonance coupling," 2016 Progress in Electromagnetic Research Symposium (PIERS), Shanghai, 2016, pp. 2941-2945 doi:10.1109/PIERS.2016.7735162

[22] V. Kindl, T. Kavalir and R. Pechanek, "Key construction aspects of low frequency wireless power transfer system using parallel resonance," 2015 17th European Conference on Power Electronics and Applications (EPE'15 ECCE-Europe), Geneva, 2015, pp. 1-5. doi:10.1109/EPE.2015.7311758

[23] C. Xu, K. Dai, X. Chen, L. Peng, Y. Zhang and Z. Dai, "Parallel Resonance Detection and Selective Compensation Control for SAPF With Square-Wave Current Active Injection," in IEEE Transactions on Industrial Electronics, vol. 64, no. 10, pp. 8066-8078, Oct. 2017 doi:10.1109/TIE.2017.2696461

[24] W. Yeetum and V. Kinnares, "Parallel Active Power Filter Based on Source Current Detection for Antiparallel Resonance With Robustness to Parameter Variations in Power Systems," in IEEE Transactions on Industrial Electronics, vol. 66, no. 2, pp. 876-886, Feb. 2019. doi:10.1109/TIE.2018.2831187

[25] Z. Shuai, D. Liu, J. Shen, C. Tu, Y. Cheng and A. Luo, "Series and Parallel Resonance Problem of Wideband Frequency Harmonic and Its Elimination Strategy," in IEEE Transactions on Power Electronics, vol. 29, no. 4, pp. 1941-1952, April 2014 doi:10.1109/TPEL.2013.2264840

[26] J. Penttonen, M. Lehtonen and S. Muhammad, "Smart grid element: efficient controllable inductance with virtual air gap," in IET Generation, Transmission \& Distribution, vol. 12, no. 1, pp. 72-77, 2 1 2018. doi:10.1049/iet-gtd.2016.2122 